\title{
Núcleo de gestão da informação e inteligência da Escola Superior da Defensoria Pública do Estado da Bahia ${ }^{1}$
}

\author{
Barbara Coelho Neves \\ Universidade Federal da Bahia - Brasil
}

ESTUDOS / CASES

\begin{abstract}
Resumo
Esta comunicação apresenta o relato de experiência acerca da implementação do Núcleo de Gestão da Informação e Inteligência administrado pela Biblioteca da Defensoria Pública do Estado da Bahia. O Núcleo tem o propósito de corroborar com o estoque informacional, baseando-se na captação do conhecimento tácito dos Defensores Públicos da DPE. Ambiciona-se a inclusão social e preservação da memória dos direitos humanos empregada nas atividades defensoriais desenvolvidas. $O$ maior benefício da criação do Núcleo de Gestão da Informação e Inteligência é proporcionar a disponibilidade de informação adquirida pelos Defensores, de maneira estruturada, a todas as pessoas interessadas nos temas defensoriais apreendidos através de vivências jurídicas, congressos, cursos de capacitação, seminários, workshops, dentre outros. O Núcleo de Gestão da Informação e Inteligência da Biblioteca da DPE constitui importante meio para conectar eficientemente "aqueles que sabem" com aqueles que "necessitam saber" e converter conhecimento pessoal em memória da organização. Para tanto, a proposta desenvolve a coleta, o armazenamento, gerenciamento e disseminação do conhecimento com uma metodologia baseada em modelos dos autores Nonaka e Takeuchi, com o auxílio de adolescentes "menores aprendizes" em cumprimento de medida sócio-educativa da Fundação Cidade Mãe.
\end{abstract}

Palavras-chave

Gestão da informação; Biblioteca especializada; Defensoria pública; Inclusão social

\section{Center for information management and intelligence of the School of Public Legal Defense of the State of Bahia}

\begin{abstract}
This paper presents the experience report about the implementation of the Center for Information Management and Intelligence administered by the Library of the Public Defender of the State of Bahia. The Center aims to corroborate the information stock, based on capturing the tacit knowledge of public Defenders of DPE. Aspires to social inclusion and the preservation of memory used in human rights activities defensoriais developed. The biggest benefit of creating the Center for Information Management and Intelligence is to provide the availability of information gained by the defenders in a structured way, to all persons interested in the topics defensoriais seized through legal experiences, conferences, training courses, seminars, workshops, Among others. The Center for Information Management and Intelligence of the DPE Library is an important way to efficiently connect "those who know" with those who "need to know" and converting personal knowledge into the organization's memory. For this, the proposal develops the collection, storage, management and dissemination of knowledge with a methodology based on models of the authors Nonaka and Takeuchi, with the help of teenagers "apprentices" in fulfillment of socio-educational measure the Mother City Foundation.
\end{abstract}

Keywords

Information management; Specialized library; Public defender; Social inclusion

\footnotetext{
${ }^{1}$ Comunicação proveniente do Projeto Gestão da Informação e Inteligência da ESDEP, ganhador do prêmio Ideias e Ações Inovadoras Governo do Estado da Bahia em 2010.
} 


\section{Introdução}

A presente comunicação consiste na síntese de uma proposta de implementação do Núcleo de Gestão da Informação e Inteligência para a Escola Superior da Defensoria Pública do Estado da Bahia (ESDEP), integrante dos Órgãos Auxiliares da Defensoria Pública do Estado (DPE).

A ESDEP desenvolve um trabalho que contribui com a difusão da informação por meio do desenvolvimento de atividades que têm como finalidade promover o aperfeiçoamento profissional e cultural do quadro de pessoal da DPE.

Desse modo, a ESDEP confere cursos de atualização, atividades de capacitação funcional, além de estimular 0 intercâmbio com organizações oficiais e entidades da sociedade civil. Sua missão compreende, como um de seus principais aspectos, o estímulo à preparação da carreira de seu quadro profissional, visando à disposição de informações atualizadas para o desenvolvimento profissional e institucional. Somente entre os anos de 2009 e 2010, a ESDEP estimulou a participação de mais de 100 membros e de 40 servidores em mais de 30 eventos em âmbito nacional e internacional. Estes beneficiários participam de palestras, cursos, congressos, workshops, dentre outros, criando uma rede de relacionamentos (network) e de contextos de apreensão de conteúdos informacionais sobre as áreas defensoriais e afins.

A gestão da informação e do conhecimento implica em adoção de práticas gerenciais compatíveis com as conclusões citadas sobre os processos de criação e aprendizado individual e, também, na coordenação sistêmica de esforços em vários planos: organizacional; estratégico e operacional; normas formais e informais. (TERRA, 2001 apud MOLINA, 2008, p. 78).

Atualmente, o volume de informações cresce a uma grande velocidade, fazendo com que haja certa dificuldade em organizar e disponibilizar todas essas informações. Existe a necessidade de se difundir rapidamente o maior volume possível de informações em cada área. A gestão da informação é um meio de coletar, organizar e disseminar esse conhecimento gerado. Analisando nessa ótica, se fez necessária a implementação de um serviço de gestão da informação, onde se possa desenvolver, de maneira estratégica, a coleta, armazenamento e disseminação do estoque de informações dos Defensores Públicos e demais beneficiários pelas atividades desenvolvidas pela ESDEP. A outra frente, que esta comunicação também vislumbra, é o tocante a inclusão social que o projeto visa proporcionar. Pois, além de garantir que todos tenham acesso às informações, envolve o treinamento e qualificação dos menores aprendizes da Fundação Cidade Mãe, em cumprimento de atividades sócio-educativas na DPE. Como resultado, apresento aos pares algumas observações dessa experiência que acredito corroborar com o estoque informacional baseado na capitação do conhecimento tácito da DPE.

Assim, essa comunicação relata os principais pontos que fundamentaram o projeto de desenvolvimento do Núcleo de Gestão da Informação e Inteligência da Escola Superior da Defensoria Pública do Estado da Bahia, ambientado na Biblioteca da ESDEP, com o propósito de divulgar essa proposta de convergência entre gestão da informação e inclusão social.

\section{Gestão da informação e inclusão social}

Constitui uma das propostas principais da ESDEP a criação de um ambiente que vise à organização e 0 atendimento da demanda informacional intra e extra-institucional concernente no acesso aos documentos impressos, digitais e virtuais sobre os temas de interesse da área jurídica e afins. O entendimento da associação entre gestão da informação e inclusão social garante a relevância e continuidade do projeto.

O maior benefício da criação do Núcleo de Gestão da Informação e Inteligência é proporcionar a disponibilidade da informação adquirida pelos beneficiários a todas as pessoas interessadas nos temas defensoriais. Trata-se de disseminar para todos os membros da DPE, aspectos do conteúdo e principais ideias que os beneficiados tiveram acesso através de congressos, cursos de capacitação, vivências jurídicas, seminários, workshops, dentre outros, constantemente viabilizados pela Instituição. Os ambientes que se propõem gerir informação constituem importantes para conectar eficientemente "aqueles que sabem" com aqueles que "necessitam saber" e converter conhecimento pessoal em conhecimento da organização. 
Desse modo, que se justifica a criação do Núcleo de Gestão da Informação e Inteligência da ESDEP, respaldandose no cenário atual que envolve as instituições públicas, que vislumbram o aprender a aprender e na possibilidade de tornar explicito e codificado os processos tácitos individuais e coletivos de construção de conhecimento no ambiente organizacional (MOLINA, 2008), ou seja, criar condições para que o conhecimento aflore e seja partilhado em função do interesse de todos e da missão da organização.

Com base no comentário de Thomas Stewart, na revista Fortune em 1991, o conhecimento das organizações é a soma de tudo que as pessoas sabem e é essa inteligência que confere a vantagem competitiva. Contudo, também as instituições públicas acumulam conhecimentos na mente das pessoas e informações de forma não estruturadas. E embora que diante da superestrutura (Estado) as organizações tenham objetivos diferentes das instituições públicas, entende-se, concordando com Molina (2008) que o desenvolvimento de práticas de gestão da informação propicia a configuração de ambiente de aprendizado e compartilhamento. A gestão da informação trabalha com a perspectiva de coleta, validação, avaliação e aplicação.

O modelo de gestão do conhecimento de Nonaka e Takeuchi (1995) vem sendo bastante difundido em projetos de gestão da informação de grandes organizações brasileiras, a exemplo da Petrobras. Com sucesso, essas empresas vêm se utilizando das categorias SECI elaboradas pelos autores para estabelecer complexos sistemas de gestão da informação com base na divulgação do conhecimento.

Desse modo, o projeto do Núcleo de Gestão da Informação e inteligência da ESDEP tem como fundamentação as categorias de compreensão da espiral do conhecimento de Nonaka e Takeuchi (1995), entendendo que a criação do conhecimento é um processo contínuo de interações dinâmicas entre o tácito e o explicito.

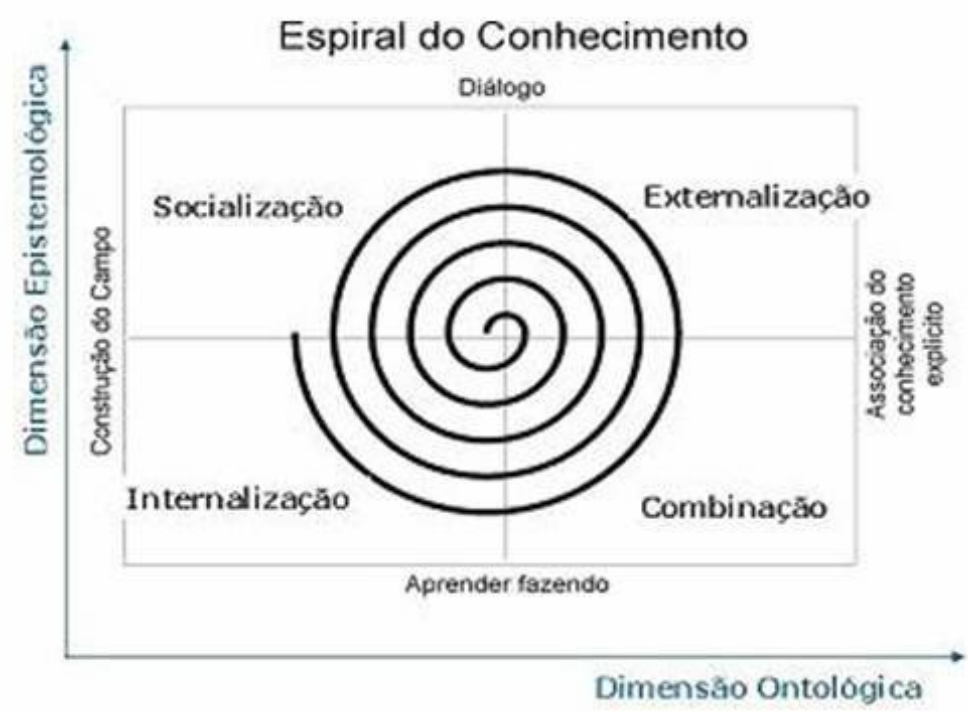

Figura 1 - SECl (Nonaka e Takeuchi, 1995).

$\mathrm{Na}$ socialização acontece o compartilhamento do conhecimento tácito, sendo a experiência compartilhada; na externalização os conceitos são identificados e combinados; na combinação as informações são organizadas; e na internalização é a possibilidade do momento de catarse, onde o "outro" pode desenvolver novos conhecimentos ao ter acesso a essas informações. (NONAKA; TAKEUCHI, 1995). Compreende-se que estas quatro modalidades da conversão do conhecimento interagem em forma de espiral, sendo relevante para introduzir na realidade da Defensoria Pública o entendimento que a socialização da informação, externalizada, estruturada e organizada contribui como mais uma variável da missão educadora da ESDEP, aumentando gradativamente o conhecimento da instituição DPE.

A gestão da informação, embora condicione diretamente em seus objetivos e conceitos o direcionamento às instâncias intelectuais de pessoas e organizações, também pode ser compreendida como uma maneira de contribuir 
com a inclusão. Nessa perspectiva, entende-se que a gestão da informação se utilizada para compartilhar, divulgar e disseminar informações, que até então eram de conhecimento de poucos, acredita-se que proporciona a inclusão de maiores parcelas do público, criando um coletivo.

O acesso à informação sempre esteve ligado à inclusão social, como pontuam Burke e Ornstein (1998), relatando que os ancestrais do homem moderno usaram, desde os primeiros assentamentos agrícolas, passando pela criação dos numerais e do alfabeto, o conhecimento sobre técnicas já dominadas, visando manter, reforçar e centralizar o domínio sobre a sociedade, beneficiando a um pequeno grupo. Durante todo este tempo,

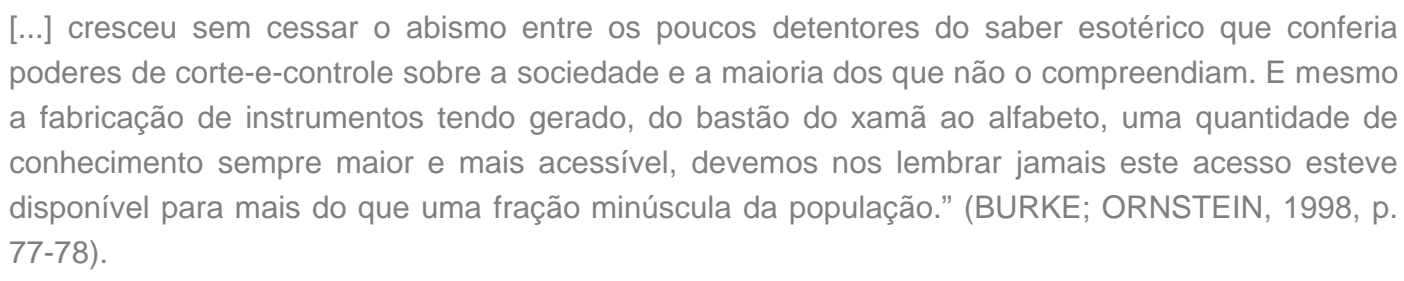

As informações e os conhecimentos restritos a poucas pessoas terminam por alimentar as diferenças, seja no âmbito da sociedade como no âmbito organizacional, criando um abismo. Assim, considerou-se, relevante contemplar a proposta da gestão da informação, neste projeto, visando disseminar para todos os membros da instituição e da comunidade às informações adquiridas por aqueles que tiveram acesso aos cursos, workshops, etc., disponibilizados pela Instituição. Outro aspecto que vale pontuar com referência a este projeto, trata-se da abordagem dada a sua metodologia de concepção. O viés da inclusão social também foi contemplado com a proposta de se treinar os adolescentes "menores aprendizes" em cumprimento de medida sócio-educativa, oriundos da Fundação Cidade Mãe, para desenvolver a coleta das informações com os membros beneficiados.

\section{Metodologia}

O Núcleo de Gestão da Informação e Inteligência da ESDEP visa atender um público alvo formado pelos defensores da sua Sede em Salvador e das 5 (cinco) regionais - Feira de Santana, Itabuna, Ilheus, Vitória da Conquista, Santo Antônio de Jesus - que atendem à 34 comarcas.

Desse modo, o público real do Núcleo compreende (os Defensores Públicos e os servidores) e o público potencial compreende (os estagiários de nível superior, os estagiários de nível médio e os interessados em seu acervo).

Destacam-se dois tipos de conhecimento na organização: Explicito: transmitido por meio de linguagem formal; Tácito: transmitido através de exemplos e da convivência.

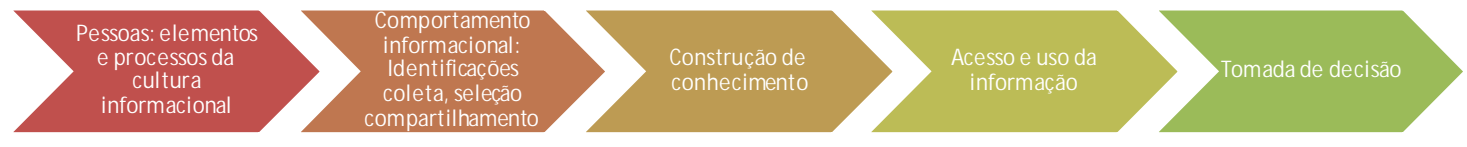

Figura 2 - Fluxo proposto de gestão da informação.

Para alcançar os objetivos deste projeto foi necessário seguir alguns passos com base em uma metodologia de trabalho, procurando seguir a linha de Nonaka e Takeuchi (1995).

\section{- Passo 1:}

- Preparar formulários de coleta de informação;

- Treinar os menores aprendizes para o processo de coleta; 


\section{Biblios center for information management and intelligence}

- Passo 2:

- Processos de transmissão do conhecimento:

- Combinação (explicito para explicito);

- Internalização (explicito para tácito);

- Socialização (tácito para tácito);

- Externalização (tácito para explicito).

- Processo de coleta:

- Gravação da informação/conhecimento.

- Processamento técnico: tombamento; classificação, catalogação;

- Etiquetagem eletromagnética e código de barra do sistema;

- Preparo técnico de cada unidade de informação para colocar na estante, disponibilizando-a para o público.

\section{Considerações finais}

A gestão da informação vem apresentando excelentes resultados em grandes organizações, que pretendem universalizar as informações e com isso manter um ambiente informacionalmente integrado.

De maneira direta e objetiva, visa-se com o Núcleo de Gestão da Informação e Inteligência da Escola Superior da Defensoria Pública proporcionar conhecimento tácito (dos membros da DPE - que participam de eventos) em explicito, colaborando com a inteligência organizacional da Defensoria Pública do Estado. Para tanto, coleta-se as informações dos membros e servidores que participam dos eventos viabilizados pela ESDEP; armazena-se o conteúdo em mídias alocadas na Biblioteca da ESDEP, possibilitando o acesso daqueles que não participaram da atividade (evento, congresso, etc.); gerencia-se a informação de modo a sistematizá-la e torná-la disponível ao público de usuários da Biblioteca da ESDEP; para posterior disseminação do conhecimento (antes tácito), agora de forma explicita, proporcionando socialização e fortalecimento da missão da ESDEP.

Para concluir, cumpre assinalar que esta comunicação tem o intuito de explicitar a proposta do Núcleo e alguns aspectos de sua fundamentação. Vale ainda salientar que não se teve a intenção de exaurir toda a contextualização que norteou este projeto que foi aprovado em âmbito estadual e agraciado com o prêmio Ideias e Ações Inovadoras da Defensoria Pública. 


\section{Referências}

MOLINA, L. G. Gestão da informação e do conhecimento e as TICs aplicadas aos portais corporativos. VALENTIM, M. Gestão da informação e do conhecimento. São Paulo: Polis, 2008.

NEVES, B. C. Projeto Sistema de Informação PT-BA. Salvador, 2009.

NEVES, B. C. Projeto CDI Rede Pintadas. Pintadas, 2008.

NONAKA, I.; TAKEUCHI, H. The knowledge-creating company. New York: Oxford University Press, 1995.

STEWART, Thomas A. The leading edge: intellectual capital. Fortune Magazine, 1991.

\section{Dados da autora}

Barbara Coelho Neves

Doutoranda em Educação (UFBA). Mestrado em Ciência da Informação (PPGCI-UFBA). Graduada em Biblioteconomia e Documentação (ICI-UFBA), possuindo especialização em Gestão da Comunicação Organizacional Integrada (NPGA-UFBA). Colunista de Tecnologia e Informação da Rede do Jornal Brasil Diário.

barbaracoelho2000@yahoo.com.br

Recebido - Received: 2011-05-26

Aceito - Accepted: 2012-03-31

\section{(cc) EY-NC-ND}

This work is licensed under a Creative Commons Attribution-Noncommercial-No Derivative Works 3.0 United States License.

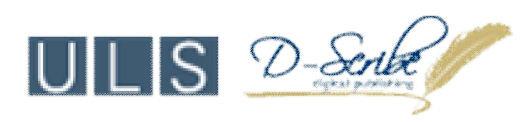

This journal is published by the University Library System of the University of Pittsburgh as part of its D-Scribe Digital Publishing Program and is cosponsored by the University of Pittsburgh Press. 